\title{
Solitary Fibrous Tumor of the Orbit: A Clinicopathologic Study of Two Cases With Review of the Literature
}

\author{
MIZUHO MITAMURA ${ }^{1,2}$, SATORU KASE ${ }^{1}$, YASUO SUZUKI ${ }^{2}$, TAKATOSHI SAKAGUCHI ${ }^{2}$, YUKA SUIMON ${ }^{1}$, \\ YOKO DONG ${ }^{1}$, KANAKO C. HATANAKA $^{3,4}$, TOSHIYA SINOHARA $^{5}$, MANABU KASE $^{2}$ and SUSUMU ISHIDA ${ }^{1}$ \\ ${ }^{1}$ Department of Ophthalmology, Faculty of Medicine and Graduate School of Medicine, \\ Hokkaido University, Sapporo, Japan; \\ ${ }^{2}$ Department of Ophthalmology, Orbital Disease \& Neuro-Ophthalmology Center, \\ Teine Keijinkai Hospital, Sapporo, Japan; \\ ${ }^{3}$ Department of Surgical Pathology, Hokkaido University Hospital, Sapporo, Japan; \\ ${ }^{4}$ Clinical Biobank, Clinical Research and Medical Innovation Center, \\ Hokkaido University Hospital, Sapporo, Japan; \\ ${ }^{5}$ Department of Pathology, Teine Keijinkai Hospital, Sapporo, Japan
}

\begin{abstract}
Background/Aim: Orbital solitary fibrous tumor (SFT) is a rare lesion among orbital tumors, which can be misdiagnosed as another mesenchymal tumor. In this study we report two cases of orbital SFT, focusing on the imaging and pathological findings of the vascular structure inside the tumor. Case Report: A 26-year-old woman and 43-year-old man presented with orbital SFT. The pathological findings revealed a patternless growth pattern of the tumor cells and hemangiopericytoma-like vascularity as well as thickened, dilated blood vessels within the tumor tissue. Tumor cells revealed a diffuse strong positivity for cluster of differentiation 34 (CD34) and signal transducer and activator of transcription 6 (STAT6) in both cases, while Bcell lymphoma 2 (bcl-2) and CD99 were positive in one case. Characteristic findings within the tumor were the arterial components, where a variety of STAT6, CD99 and bcl-2positive smooth muscle cells were intermingled. Conclusion: Histologically, the tumor tissues might be characterized by not only conventional hemangiopericytoma-like vasculature but also dilated arterial vessels, which were shown to be part of the tumor components.
\end{abstract}

This article is freely accessible online.

Correspondence to: Satoru Kase MD, Ph.D., Department of Ophthalmology, Faculty of Medicine and Graduate School of Medicine, Hokkaido University, N-15, W-7, Kita-ku, Sapporo 0608638, Japan. Tel: +81 117065944, Fax: +81 117065948, e-mail: kaseron@med.hokudai.ac.jp

Key Words: Mesenchymal tumor, orbital tumor, solitary fibrous tumor, feeding artery.
Solitary fibrous tumor (SFT), a rare spindle-cell tumor, commonly arises from the pleura (1) but may also occur in extrapleural sites (2-5). Orbital SFT is a rare lesion and about 90 cases have been reported in the literature to date $(6,7)$. This tumor is likely to simulate other mesenchymal tumors such as fibrous histiocytoma, liposarcoma, synovial sarcoma, and neurofibroma. Since SFT is rich in feeding arteries, preoperative clinical diagnosis is important in assessing the likelihood of intraoperative bleeding (8), and total tumor resection is recommended (9). However, histopathological findings of the feeding vessels in SFT remain unknown. In this study, we report two cases of orbital SFT, focusing on the imaging and pathological findings of the vascular structure inside the tumor. The institutional review board in Hokkaido University and Teine Keijinkai Hospital waived the ethical assessment of the clinical study because of case reports. This study adhered to the principles of the Declaration of Helsinki.

\section{Case Report}

Case 1. A 26-year-old woman presented with progressive and painless lower eyelid swelling for 2 months. An elastic hard, non-tender and non-pulsatile mass was palpable in the lower eyelid. Computed tomography revealed a well-defined heterogeneous mass along the lower wall of the right orbit (Figure 1A). Magnetic resonance imaging (MRI) demonstrated an orbital lesion with an isointense signal on T1-weighted images (T1WI) (Figure 1B) and a hyperintense signal on T2weighted images (T2WI) (Figure 1C). Postcontrast T1WI revealed a strong enhancement of the lesion that contained a linear flow void-like hypointensity (Figure 1D, arrow). Surgical excision was performed. There was bleeding from the 

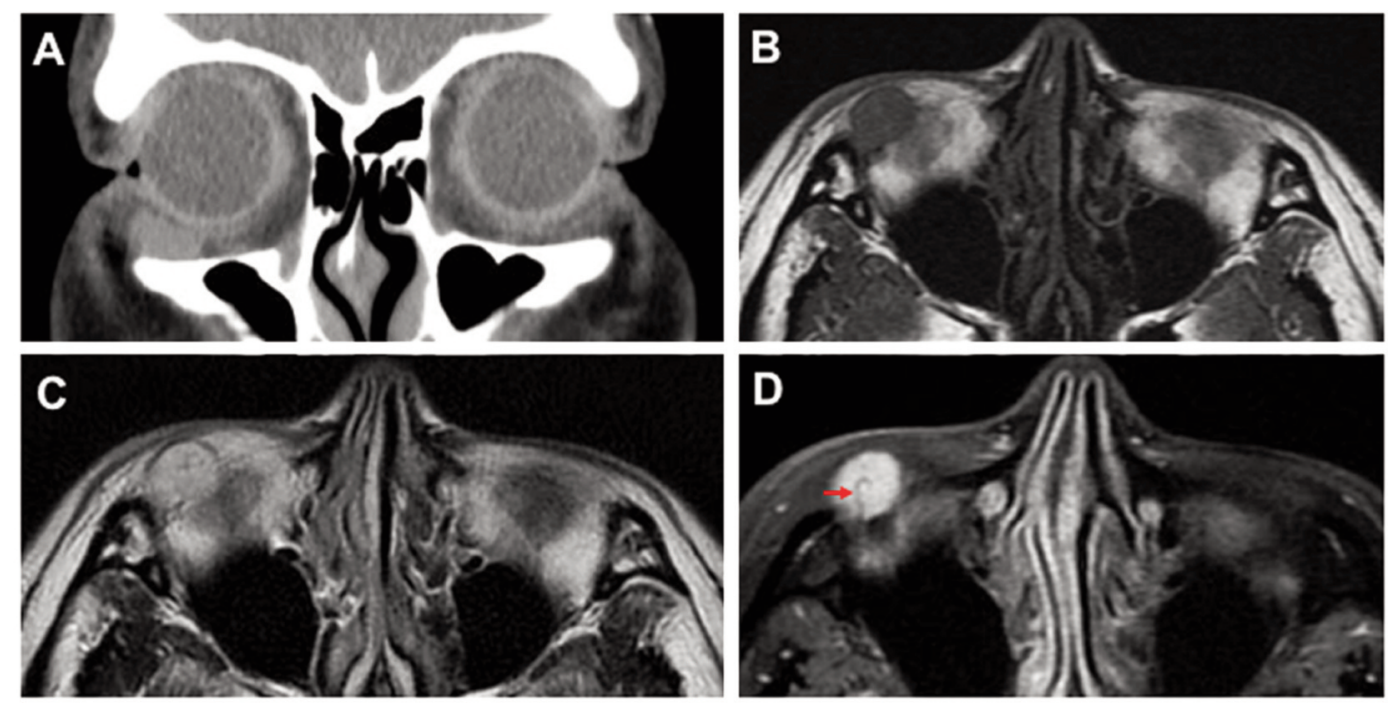

Figure 1. Computed tomography (CT) and axial images of magnetic resonance imaging (MRI) in Case 1. (A) CT showing a well-defined heterogeneous mass along the lower wall of the right orbit. (B) Non-contrast T1-weighted image (T1WI) showing an orbital lesion of isointense signal. (C) Noncontrast T2-weighted image (T2WI) showing an orbital lesion of hyperintense signal. (D) Postcontrast T1WI revealed enhancement of the lesion containing linear flow void-like hypointensity (arrow).

inflowing blood vessel when the tumor was detached, suggesting the tumor had high vascularity. The postoperative period was uneventful, and no recurrence was observed at the time of last examination 5 months postoperatively.

Pathological findings in Case 1. Gross examination showed an oval reddish mass measuring $13 \times 10 \times 8 \mathrm{~mm}$. The lesion had a thin fibrous cap, and a mixture of coarse and dense cells was observed named "patternless growth pattern" (Figure 2A). In the dense part, cells with round to short fusiform nuclei could be identified. In the sparse part, similar cells were found on a myxoid background together with multinucleated cells (Figure 2B, C). The vasculature called "staghorn configuration" was inconspicuous.

Immunohistochemistry showed that tumor cells were strongly positive for CD34 (Figure 2D), signal transducer and activator of tracscription6 (STAT6) (Figure 2E, F), $\beta$-catenin (nuclear staining) and the progesterone receptor (PgR) (partial nuclear staining). However, immunoreactivity for B-cell lymphoma 2 (bcl-2), CD31, S100 protein, SOX-10, desmin, $\alpha$ smooth muscle actin ( $\alpha \mathrm{SMA})$, myogenin, human melanin black 45 (HMB45), Wilms' tumor gene product 1 (WT1), epithelial membrane antigen (EMA), and CD99 was negative. Negative results on bcl-2 immunoreactivity made it difficult to distinguish it from other tumors; however, all reported cases with SFT did not reveal bcl-2-positivity (10). Instead of bcl-2 immunoreactivity, based on the recent consensus on diagnostic contribution of STAT6 (11-15), immunohistochemical features showing diffuse nuclear STAT6-positive tumor cells were used to establish a diagnosis of orbital SFT.
Case 2. A 43-year-old man was referred to our clinic because of blepharoptosis, proptosis and downward displacement of the eyeball for the past year. An elastic hard, non-tender, non-pulsatile mass was palpable under the right upper eyebrow. T1WI and T2WI MRI revealed an isointense lesion (Figure 3A), and a heterogeneous lesion with linear flow void-like hypointensity (Figure 3B, arrow), respectively. Postcontrast T1WI revealed an enhancement corresponding to the supraorbital artery in the contralateral eye (Figure 3C, arrow). A hyperintense lesion was considered to be a blood vessel flowing into the tumor (Figure 3D, arrow). Surgical excision was performed. The tumor was multinodular and rich in blood vessels. The postoperative period was uneventful, and no recurrence was observed at the time of the last examination, 5 years after surgery.

Pathological findings in Case 2. Gross examination showed an slightly shiny grayish white mass measuring $23 \times 20 \times 17$ $\mathrm{mm}$ (Figure 4A). The lesion had a fibrous cap surrounding it. Internal hollow structures were observed, which were thought to be feeding vessels (Figure 4A, arrows). The cell density varied within the tumor tissue; a slightly lower cell density was intermingled with myxomatous backgrounds (Figure 4B, D). Although the vasculature called "staghorn configuration" was not clearly visible in this case, the dilated vessel exhibited endothelial cells lining the lumen and abundant stratified spindle cells surrounding the lumen. The vascular configuration corresponded to the inflow of the arterial component shown in MRI. 

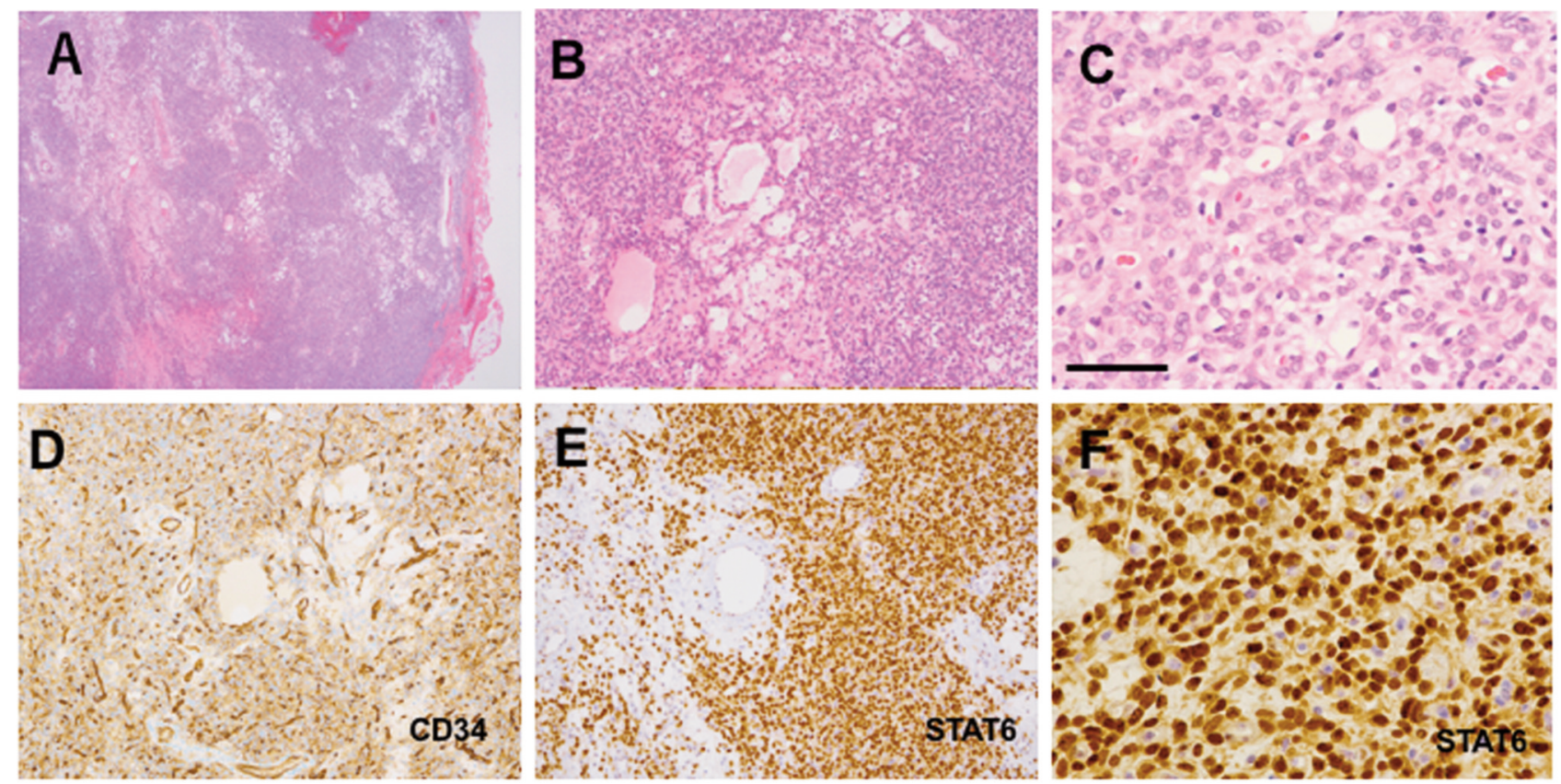

Figure 2. Pathological and Immunostaining Findings in Case 1. (A) The lesion had a thin fibrous cap and a mixture of coarse and dense cells, a so-called "patternless growth pattern" [Hematoxylin and Eosin (H\&E), 40x magnification]. (B) The tumor tissue contained myxomatous stroma (H\&E, 200x magnification). (C) A higher magnification image revealed a collection of tumor cells with small vessels (H\&E, 400× magnification). Bar indicates $50 \mu \mathrm{m}$. (D) Cluster of differentiation 34 (CD34) immunostaining was positive in the stroma (200x magnification). (E) Signal transducer and activator of tracscription6 (STAT6) immunopositive nuclei were noted in the tumor tissue (200x magnification). (F) STAT6 positivity was identified in the nuclei of tumor cells (400x magnification).
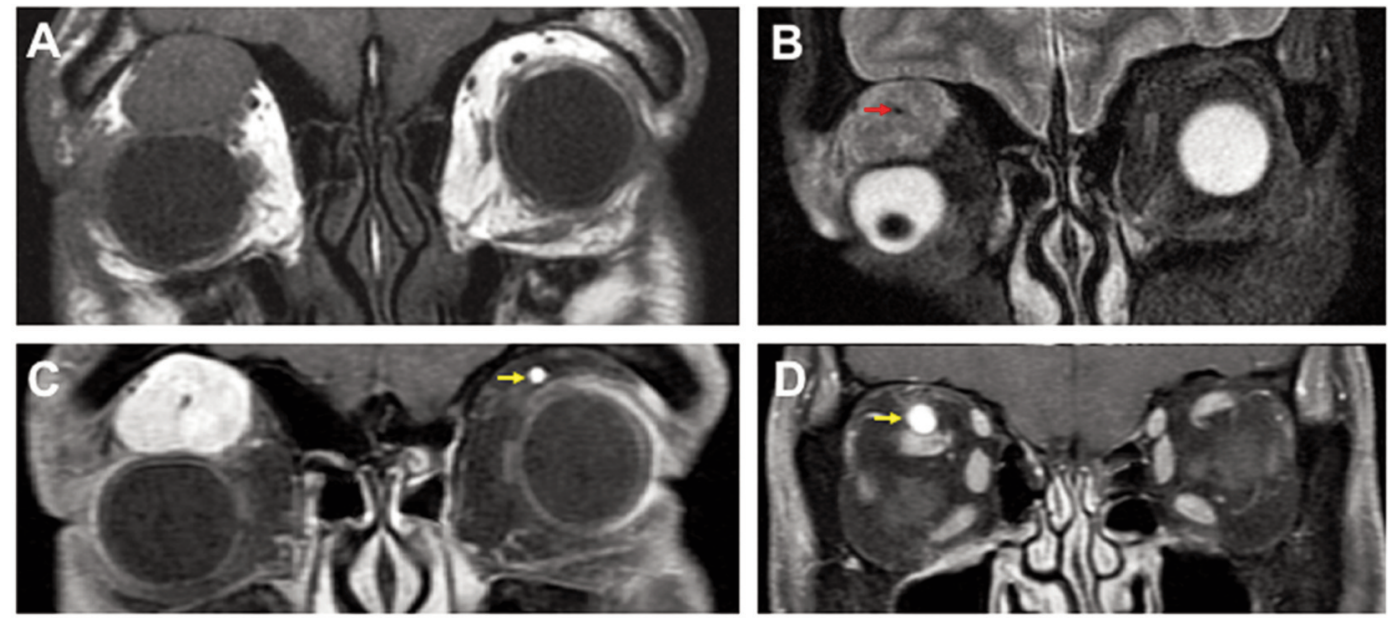

Figure 3. MRI in Case 2. (A) Noncontrast T1WI showing a well-defined, lobulated mass of isointense signal. (B) Noncontrast T2WI showing a heterogeneous lesion containing linear flow void-like hypointensity (arrow). (C) Postcontrast T1WI revealed an enhancement of the lesion corresponding to the supraorbital artery in the opposite eye (arrow). (D) A hyperintense lesion considered to be a blood vessel flowing into the tumor was observed (arrow).

Immunohistochemistry showed tumor cells to be positive for CD34, bcl-2, CD99, and STAT6 (Figure 4C) and negative for S100 protein, EMA, glucose transporter 1, factor XIIIa and $\alpha$ SMA. Furthermore, we looked into immunoreactivity of the inflow of arterial components. The arterial components were CD34-positive in the vascular endothelial cells, but negative in the surrounding spindle cells (Figure 4E). Bcl-2 was positive in the spindle cells (Figure 4F), 

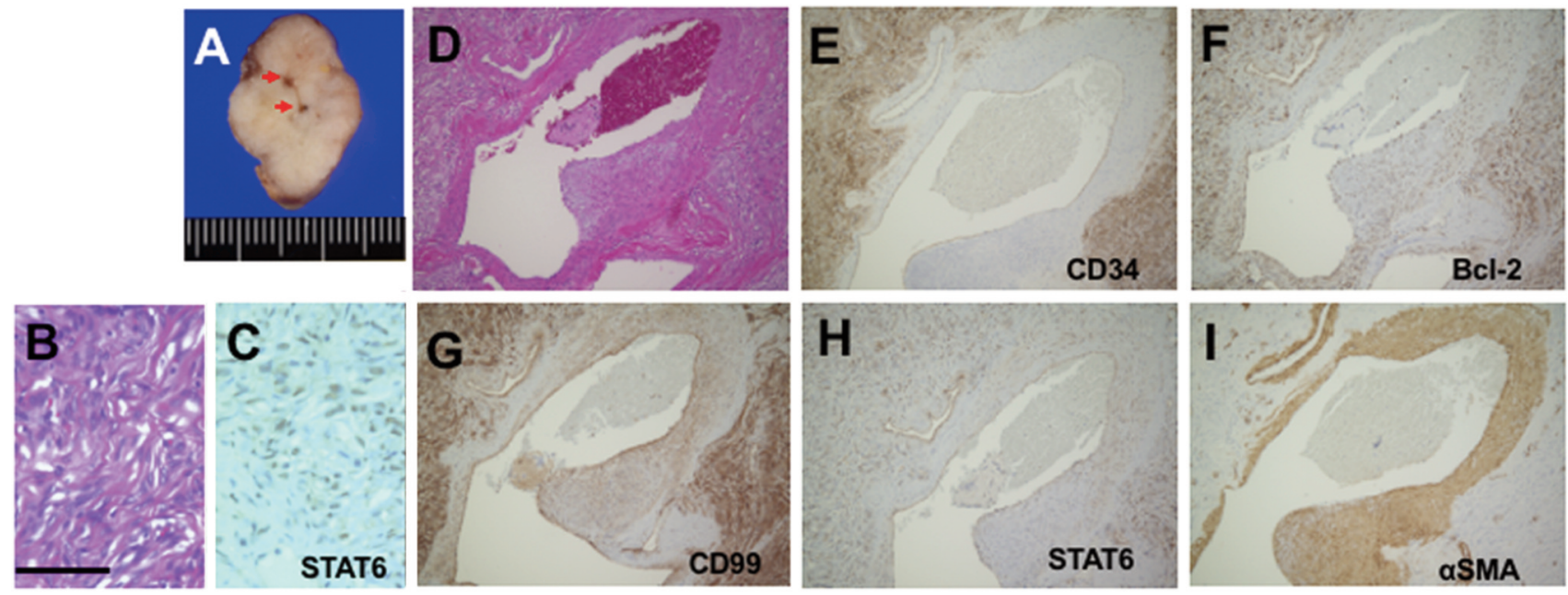

Figure 4. Pathological and immunohistochemical findings in Case 2. Gross examination showed two hollow structures, indicating feeding vessels (arrows). High magnification revealed spindle tumor cells (H\&E, 400x magnification). A bar indicated 50 um. Immunoreactivity for STAT6 was observed in the nuclei (400x magnification). (D) Spindle cells showed "patternless growth pattern". The dilated vessel and abundant stratified spindle cells were observed (H\&E, 100x magnification). (E) CD34 immunostaining was positive in the stroma and vascular endothelial cells (100x magnification). (F) B-cell lymphoma 2 (bcl-2) was positive in the cells composing the dilated vessel (100x magnification). (G) CD99 was positive in the stroma, vascular endothelial cells and thickened vascular wall (100x magnification). (H) STAT6 was positive in the nuclei (100x magnification). (I) a smooth muscle actin was positive in the thickened vascular wall but negative in the tumor cells (100x magnification).

which was less marked than tumor cells. Immunoreactivity for CD99 (Figure 4G) and STAT6 (Figure 4H) was strongly positive in vascular endothelial cells and thickened vascular wall composed of stratified spindle cells. $\alpha$ SMA was positive in the spindle cells (Figure 4I), indicating smooth muscle cells composing the dilated vessel. Taken together, these morphological and immunohistochemical features were consistent with orbital SFT.

\section{Discussion}

The pathological findings of our cases showed a typical "patternless growth pattern" which is characterized by bland spindle-cell proliferation alternating hyper- and hypo-cellular areas and a focal hemangiopericytoma-like vascular pattern (5). The diagnosis of SFT can be solidified by the use of immunohistochemical analysis. SFT shows immunopositivity for CD34, vimentin, bcl-2, CD99, STAT6, and early growth response protein 1, but is negative for cytokeratin, EMA, S100 protein, $\alpha$ SMA, factor VIII-related antigen and desmin (1214, 16-19). Tumor cells in our cases were strongly positive for CD34 and STAT6 in both cases, while bcl-2 and CD99 were positive in Case 2. In contrast, immunoreactivity for S-100 protein, $\alpha$ SMA and EMA was not detected in tumor cells.

Compared with the cerebral cortex or muscle, MRI basically depicts isointensity and isointensity to hypointensity on T1WI and T2WI, respectively, although a few lesions may show heterogeneous hyperintensity or cystic appearance in $\operatorname{SFT}(5,16,20)$. It is known that signal intensity-void, tubular structures are shown on MRI, which represent fast-flow vessels within the tumor $(5,16,20)$. This was seen in both our cases. In the preoperative image and intraoperative findings of our cases, linear flow voids and fast-flowing blood vessels in the tumor suggest a blood flowrich tumor, which are unusual features in hemangiomas. These data also indicate that orbital biopsy should be strictly prohibited to avoid serious intraoperative bleeding (8).

The vascular structure of SFT is known to be of a "staghorn configuration" and/or "hemangiopericytoma-like vasculature". These characteristics are rich in irregular, angular, small vascularity with thin-walled, branching blood vessels and frequently hyalinized, thick-walled vessels (15, 21). In our cases, tumor tissues in Case 2 posed a typical hemangiopericytoma-like pattern, whereas dilated vessels with a thick vascular wall were exclusively composed of aSMA-positive smooth muscle cells. Since the "staghorn configuration" is characterized by a thin vessel wall that is aSMA-negative and lacking smooth muscle cells $(11,15$, 22), the blood vessels observed in Case 2 could be smooth muscle cell-rich vascular components.

This study further focused on the immunoreactivity for STAT6, CD99, and bcl-2 in the dilated vessels in Case 2. The characteristic finding was the inflow of smooth-muscle cellrich vascular components, accompanied by a large number of STAT6, CD99 and bcl-2-positive smooth muscle cells. There is no previous report focusing on the immunostaining of 
vascular structures inside tumors, but comparing the previous reports on immunohistochemical expression of SFT, "staghorn" blood vessels has been considered negative for STAT6 and bcl-2 $(11,15,22)$. From the above, it is shown that these dilated blood vessels with thickened walls composed of smooth muscle cells show different histological features from "staghorn" blood vessels, based on the immunohistochemical results. Macroscopic pathological findings indicate that this blood vessel is consistent with a feeding blood vessel. The vascular structure was microscopically shown to be part of the tumor, and the constituting cells might have differentiated from mesenchymal tumor cells. However, the significance of STAT6 and bcl-2 positivity in smooth muscle cells constituting the blood vessel wall is unknown, and further research is needed. Moreover, another limitation of this study is that it could not examine elastic fibers or other markers of smooth muscle cells.

In conclusion, orbital SFT vessels were characterized by conventional hemangiopericytoma-like vasculature as well as intratumoral dilated vessels, consistent with feeder vessels. The vascular configuration was characterized immunohistochemically. Since biopsy procedures may cause serious intraoperative bleeding, clinical diagnosis by preoperative MRI findings and total tumor resection are strongly recommended according to clinicopathological findings.

\section{Conflicts of Interest}

The Authors declare no conflicts of interest regarding this study.

\section{Authors' Contributions}

MM corrected clinical data and wrote the paper. SK, KH and TS acquired pathological findings and interpreted the clinicopathological data. YS, TS, YS, YD, MK and SI supervised the manuscript and performed critical revisions.

\section{References}

1 Klemperer P and Rabin CB: Primary neoplasmas of the pleura: A case report of five cases. Am J Ind Med 22(1): 1-31, 1992. PMID: 1415270. DOI: 10.1002/ajim.4700220103

2 Brunnemann RB, Ro JY, Ordonez NG, Mooney J, El-Naggar AK and Ayala AG: Extrapleural solitary fibrous tumor: A clinicopathologic study of 24 cases. Mod Pathol 12(11): 10341042, 1999. PMID: 10574600.

3 D'Amico FE, Ruffolo C, Romano M, DI Domenico M, Sbaraglia M, Dei Tos AP, Garofalo T, Giordano A, Bassi I and Massani M: Rare neoplasm mimicking neuoroendocrine pancreatic tumor: A case report of solitary fibrous tumor with review of the literature. Anticancer Res 37(6): 3093-3097, 2017. PMID: 28551649. DOI: 10.21873/anticanres.11665

4 Bacalbasa N, Balescu I, Jinescu G, Marcu M, Contolenco A, Pop D, Dobritoiu D, Ionescu O, Ionescu P and Stoica C: Fat-forming solitary fibrous tumor of the kidney: A case report and literature review. In Vivo 32(3): 649-652, 2018. PMID: 29695573. DOI: 10.21873/invivo. 11288

5 Yang BT, Wang YZ, Dong JY, Wang XY and Wang ZC: MRI study of solitary fibrous tumor in the orbit. AJR Am J Roentgenol 199(4): 506-511, 2012. PMID: 22997401. DOI: 10.2214/AJR.11.8477

6 Furusato E, Valenzuela IA, Fanburg-Smith JC, Auerbach A, Furusato B, Cameron JD and Rushing EJ: Orbital solitary fibrous tumor: encompassing terminology for hemangiopericytoma, giant cell angiofibroma, and fibrous histiocytoma of the orbit: reappraisal of 41 cases. Hum Pathol 42(1): 120-128, 2011. PMID: 21056898. DOI: 10.1016/j.humpath.2010.05.021

7 Shen J, Li H, Feng S, and Cui H: Orbital solitary fibrous tumor: a clinicopathologic study from a Chinese tertiary hospital with a literature review. Cancer Manag Res 10: 1069-1078, 2018. PMID: 29780261. DOI: 10.2147/CMAR.S165218

8 Demura M, Hayashi Y, Sasagawa Y, Mohri M, Takahira M and Nakada M: Intraorbital solitary fibrous tumor requiring preoperative embolization of feeding artery. Asian J Neurosurg 14(2): 593-597, 2019. PMID: 31143292. DOI: 10.4103/ajns. AJNS_30_19

9 Jackson $\mathrm{CH}$, Hunt BC and Harris GH: Fate and management of incompletely excised solitary fibrous tumor of the orbit: A case series and literature review. Ophthalmic Plast Reconstr Surg, 2020. PMID: 32496393. DOI: 10.1097/IOP.0000000000001691

10 Han Y, Zhang Q, Yu X, Han X, Wang H, Xu Y, Qiu X and Jin F: Immunohistochemical detection of STAT6, CD34, CD99 and BCL-2 for diagnosing solitary fibrous tumors/hemangiopericytomas. Int J Clin Exp Pathol 8(10): 13166-13175, 2015. PMID: 26722515.

11 Doyle LA, Vivero M, Fletcher CD, Mertens F and Hornick JL: Nuclear expression of STAT6 distinguishes solitary fibrous tumor from histologic mimics. Mod Pathol 27(3): 390-395, 2013. PMID: 24030747. DOI: 10.1038/modpathol.2013.164

12 Robinson DR, Wu YM, Kalyana-Sundaram S, Cao X, Lonigro RJ, Sung YS, Chen CL, Zhang L, Wang R, Su F, Iyer MK, Roychowdhury S, Siddiqui J, Pienta KJ, Kunju LP, Talpaz M, Mosquera JM, Singer S, Schuetze SM, Antonescu CR and Chinnaiyan AM: Identification of recurrent NAB2-STAT6 gene fusions in solitary fibrous tumor by integrative sequencing. Nat Genet 45(2): 180-185, 2013. PMID: 23313952.DOI: 10.1038/ ng. 2509

13 Chmielecki J, Crago AM, Rosenberg M, O’Connor R, Walker SR, Ambrogio L, Auclair D, McKenna A, Heinrich MC, Frank DA and Meyerson M: Whole-exome sequencing identifies a recurrent NAB2-STAT6 fusion in solitary fibrous tumors. Nat Genet 45(2): 131-132, 2013. PMID: 23313954. DOI: 10.1038/ng.2522

14 Mohajeri A, Tayebwa J, Collin A, Nilsson J, Magnusson L, Steyern FV, Brosjö O, Domanski HA, Larsson O, Sciot R, Debiec-Rychter M, Hornick JL, Mandahl N, Nord KH and Mertens F: Comprehensive genetic analysis identifies a pathognomonic NAB2/STAT6 fusion gene, non-random secondary genomic imbalances, and a characteristic gene expression profile in solitary fibrous tumor. Genes Chromosomes Cancer 52(10): 873-886, 2013. PMID: 23761323. DOI: $10.1002 / \mathrm{gcc} .22083$

15 Montes-Mojarro IA, Baquera-Heredia J, Sanchez-Marle JF and Ortiz-Hidalgo C: Immunohistochemical expression of nuclear $\beta$ catenin and STAT-6 in a solitary fibrous tumor of the soft palate: 
A case report and review of the literature. Iran J Pathol 12(3): 281-285, 2017. PMID: 29531555.

16 Kim HJ, Kim HJ, Kim YD, Yim YJ, Kim ST, Jeon P, Kim KH, Byun HS and Song HJ: Solitary fibrous tumor of the orbit: CT and MR imaging findings. AJNR Am J Neuroradiol 29(5): 857862, 2008. PMID: 18272558. DOI: 10.3174/ajnr.A0961

17 Vogels RJ, Vlenterie M, Versleijen-Jonkers YM, Ruijter E, Bekers EM, Verdijk MA, Link MM, Bonenkamp JJ, van der Graaf WT, Slootweg PJ, Suurmeijer AJ, Groenen PJ and Flucke U: Solitary fibrous tumor: clinicopathologic, immunohistochemical and molecular analysis of 28 cases. Diagn Pathol 9: 224, 2014. PMID: 25432794. DOI: 10.1186/s13000-014-0224-6

18 Dorfman DM, To K, Dickersin GR, Rosenberg AE and Pilch BZ: Solitary fibrous tumor of the orbit. Am J Surg Pathol 18(3): 281-287, 1994. PMID: 8116796. DOI: 10.1097/00000478199403000-00008

19 Westra WH, Gerald WL and Rosai J: Solitary fibrous tumor: Consistent CD 34 immunoreactivity and occurrence in the orbit. Am J Surg Pathol 18(10): 992-998, 1994. PMID: 7522416. DOI: 10.1097/00000478-199410000-00003

20 Alkatan HM, Alsalamah AK, Almizel A, Alshomar KM, Maktabi AM, ElKhamary SM, Eberhart CG, Iuliano A, Lanni V and Strianese D: Orbital solitary fibrous tumors: a multi-centered histopathological and immunohistochemical analysis with radiological description. Ann Saudi Med 40(3): 227-233, 2020. PMID: 32493043. DOI: 10.5144/0256-4947.2020.227
21 Mentzel T, Bainbridge TC and Katenkamp D: Solitary fibrous tumour: clinicopathological, immunohistochemical, and ultrastructural analysis of 12 cases arising in soft tissues, nasal cavity and nasopharynx, urinary bladder and prostate. Virchows Arch 430(6): 445-453, 1997. PMID: 9230909. DOI: 10.1007/ s004280050054

22 Ouladan S, Trautmann M, Orouji E, Hartmann W, Huss S, Büttner R and Wardelmann E: Differential diagnosis of solitary fibrous tumors: A study of 454 soft tissue tumors indicating the diagnostic value of nuclear STAT6 relocation and ALDH1 expression combined with in situ proximity ligation assay. Int $\mathbf{J}$ Oncol 46(6): 2595-2605, 2015. PMID: 25901508. DOI: 10.3892/ ijo.2015.2975
Received August 23, 2020

Revised September 6, 2020

Accepted September 8, 2020 